\title{
Exploring the global properties of Type Ia supernovae
}

\author{
Paolo Mazzali \\ Max-Planck Institute for Astrophysics, \\ Karl-Schwarzschild-Strasse 1, D-85741 Garching bei München, Germany \\ email: mazzali@mpa-garching.mpg.de
}

\begin{abstract}
While Type Ia supernovae are widely used as distance indicators, the reasons for the correlation between luminosity and light curve width that allows SNe Ia to be calibratable standard candles is not yet fully understood, and in particular the details of the explosion mechanism are still the subject of heated debate. We present the results of a systematic approach that uses the high-quality data collected by the European Research Training Network "The Physics of Type Ia Supernova Explosions" to map the supernova ejecta and to infer the properties of the explosion.
\end{abstract}

Keywords. Supernovae, photometry, spectroscopy, abundance tomography

The distribution of the elements in the outer layers of the ejecta of a (Type Ia) supernova can be derived by modelling a closely spaced time series of spectra, while the nebular spectra give information about the inner ejecta (Stehle et al. 2005). Results for SN 2002bo show that the composition stratification that is imprinted at the time of the explosion is largely preserved. Using the abundance distribution obtained as above, and assuming that all NSE elements contribute equally to the opacity while only radioactive nickel contributes to the radiative output, the light curve of SN 2002bo can be reproduced very accurately.

Examining the spectra of a large sample of SNe Ia, we find that the outer extent of the burning, as indicated by the velocity of the strong Si II $6355 \AA$ line, is approximately the same in all SNe Ia, regardless of their luminosity. We confirm that the amount and distribution of ${ }^{56} \mathrm{Ni}$ is proportional to the luminosity of the $\mathrm{SN}$, and find that SNeIa produce a roughly constant amount of stable NSE isotopes. We show that the sum of all NSE elements gives a better correlation with the width of the light curve. These results suggest that the kinetic energy of all SNe Ia is similar, and that the mass of the progenitor white dwarf is constant, which supports the single degenerate scenario.

A variation in the ratio of stable versus unstable NSE species can generate a dispersion about the mean brightness-decline rate relation. Such a variation may be the consequence of different progenitor metallicities. A systematic evolution of the metallicity of SN Ia progenitors may cause a redshift-dependent shift of SNe in the brightness-decline rate plane which may to a certain extent mimic the accelerated expansion of the Universe.

\section{Reference}

Stehle, M., Mazzali, P. A., Benetti, S., \& Hillebrandt, W. 2005, MNRAS, 360, 1231 